\title{
Determinantes da utilização de canais de serviços mobile banking para transações financeiras
}

\author{
Alexandre Corrêa dos Santos \\ Doutorando em Ciências Contábeis e Administração \\ Universidade Regional de Blumenau - FURB, SC-Brasil \\ acs@atuaris.com.br \\ http://orcid.org/0000-0003-3119-1586 \\ Marcos Paulo Albarello Friedrich \\ Doutorando em Ciências Contábeis e Administração \\ Universidade Regional de Blumenau - FURB, SC-Brasil \\ http://orcid.org/0000-0002-6579-2956 \\ marcos.ensino@gmail.com
}

Nelson Hein

Doutor em Engenharia de Produção pela Universidade Federal de Santa Catarina - UFSC, SC-Brasil Professor do Programa de Pós-Graduação em Contabilidade (Doutorado) da Universidade Regional de Blumenau - FURB, SC-Brasil

hein@furb.br

http://orcid.org/0000-0002-8350-9480

\section{Editor Científico: José Edson Lara}

Organização Comitê Científico

Double Blind Review pelo SEER/OJS

Recebido em 05.10.2019

Aprovado em 05.12.2019 


\title{
Resumo
}

Objetivo do estudo: Este estudo busca identificar quais fatores apresentam maior impacto no uso do mobile banking (MB) para movimentações financeiras.

Metodologia/abordagem: A partir de abordagem quantitativa, descritiva e documental, adotou-se a técnica estatística de Regressão Multivariada, de forma a se investigar o fenômeno por meio de estudo de caso único em uma instituição financeira brasileira, o que representou a análise de agências bancárias em 338 municípios.

Originalidade/relevância: A busca por respostas neste campo possibilita a compreensão de aspectos demográficos, que podem ser utilizados tanto para fins sociais quanto econômicos. Nesse contexto, esse estudo avança em relação aos estudos anteriores ao analisar as características dos usuários de serviços mobile banking para movimentações financeiras ao invés de somente avaliar a intenção ou uso (não financeiro) do serviço.

Principais resultados: Enquanto destaque identificou-se que indivíduos com níveis de escolaridade mais alto utilizam mais o serviço mobile banking em relação à indivíduos com níveis mais baixos de escolaridade.

Contribuições teóricas/metodológicas: $\mathrm{O}$ estudo contribui ao apontar fatores humanos que impactam no uso de mobile banking, o que avança na discussão teórica em relação a compreensão do mobile banking enquanto canal de acesso aos serviços bancários existentes ou um novo instrumento de pagamentos, ou ambos.

Contribuições Sociais/para a gestão: No campo social espera-se que este estudo aponte características de regionalidade, de forma que os investimentos em tecnologia bancária sejam mais alinhados à práticas de transformação econômica, social e ambiental.

Palavras-chave: Mobile banking; Tecnologias; Bancos.

\section{Mobile banking and the use determinants of digital channels for financial transactions}

\begin{abstract}
Study objective: This study seeks to identify which factors have the greatest impact on the use of mobile banking (MB) for financial transactions.

Methodology/approach: Based on a quantitative, descriptive and documentary approach, the statistical technique of Multivariate Regression was adopted, in order to investigate the phenomenon through a single case study in a Brazilian financial institution, which represented the analysis of bank branches in 338 municipalities.

Originality/relevance: The search for answers in this field makes it possible to understand demographic aspects that can be used for both social and economic purposes. In this context, this study advances from previous studies by analyzing the characteristics of mobile banking service users for financial movements rather than merely evaluating the intention or (nonfinancial) use of the service.
\end{abstract}


Main results: It was found that individuals with higher education levels use mobile banking more than individuals with lower education levels.

Theoretical/methodological contributions The study contributes by pointing out human factors that impact the use of mobile banking, which advances the theoretical discussion regarding the understanding of $\mathrm{MB}$ as an access channel to existing banking services or a new payment instrument, or both.

Social contributions/to management: In the social field, this study is expected to point to regional characteristics, so that investments in banking technology are more aligned with the practices of economic, social and environmental transformation.

Keywords: Mobile banking; Technologies; Banks.

\section{Servicios bancarios móviles y determinantes del uso de canales digitales para transacciones financieras}

\section{Resumen}

Objetivo del estudio: Este estudio busca identificar qué factores tienen el mayor impacto en el uso de la banca móvil (BM) para transacciones financieras.

Metodología/enfoque: Basado en un enfoque cuantitativo, descriptivo y documental, se adoptó la técnica estadística de regresión multivariante, con el fin de investigar el fenómeno a través de un único estudio de caso en una institución financiera brasileña, que representaba el análisis de sucursales bancarias en 338 municipios.

Originalidad/relevancia: la búsqueda de respuestas en este campo permite comprender los aspectos demográficos que pueden utilizarse tanto con fines sociales como económicos. En este contexto, este estudio avanza de estudios anteriores al analizar las características de los usuarios del servicio de banca móvil para los movimientos financieros en lugar de simplemente evaluar la intención o el uso (no financiero) del servicio.

Principales resultados: Si bien se destacó, se identificó que las personas con niveles de educación superior usan la banca móvil más que las personas con niveles de educación más bajos.

Contribuciones teóricas/metodológicas: el estudio contribuye al señalar los factores humanos que afectan el uso de la banca móvil, lo que avanza la discusión teórica de la comprensión de $\mathrm{MB}$, como un canal para el acceso a los servicios bancarios existentes como un instrumento de pago.

Contribuciones sociales/a la gestión: En el campo social, se espera que este estudio tenga características regionales, de modo que las inversiones en tecnología bancaria estén más estrechamente alineadas con las prácticas de transformación económica, social y ambiental.

Palabras clave: Banca móvil; Tecnologías; Bancos. 


\section{Introdução}

Nas últimas duas décadas, a tecnologia de serviços bancários migrou dos serviços de autoatendimento via caixa eletrônico (ATM) e telefone, para o internet banking (IB) e, recentemente, para o mobile banking $(\mathrm{MB})$ em um processo de virtualização em nível global que acompanhou a grande demanda de acesso à internet móvel via smartphones (Brown, et al. 2003; Shaikh, Hanafizadeh, \& Karjaluoto, 2017).

Para o Federal Reserve System (2016), a utilização do MB apresenta tendência de crescimento consistente no curto e longo prazo, pois permite aos clientes acesso em qualquer hora e lugar, oferece relacionamento mais intenso empresa-cliente e comodidades como evitar filas e trânsito, além de ter horário estendido para transações (Carlsson, Walden \& Bowman, 2006; Coelho \& Easingwood, 2003; Laukannen, 2007; Pousttchi \& Schurig, 2004, Wang, Lin, \& Luarn, 2006).

No Brasil, o número de acessos à internet nas residências por meio de aparelhos celulares acompanhou esse comportamento e supera o de acessos via computador (IBGE, 2014). Esse movimento foi acompanhado pelo setor bancário brasileiro, considerado um dos mais avançados do mundo (Ramos, Ferreira, Freitas, \& Rodrigues, 2018), no qual o MB se tornou o meio mais utilizado para transações totalizando 25,6 bilhões de operações em 2017, o que representa $35 \%$ do total do país frente aos 9,9 bilhões dos ATM e 15,8 bilhões do IB.

Diante disso, o dado que mais chama a atenção é o crescimento da utilização desse canal, pois enquanto o MB cresceu 37,63\% de 2016 para 2017, o IB cresceu apenas 1,93\% e as transações no ATM reduziram 2,94\% no mesmo período (Febraban, 2018).

No entanto, apesar do promissor potencial para a utilização do MB, a efetiva adoção dessa tecnologia para movimentações está aquém do esperado pelas instituições financeiras. Dos 25,6 bilhões de transações via mobile no Brasil, somente cerca de 6,5\% (1,7 bilhões) envolveram movimentação de recursos, as demais foram apenas para consultas de saldo e extratos (Febraban, 2018) e, esse número se repete em escala global (Funk, 2009; Shaikh, \& Karjaluoto, 2015; Suresh \& Namdeo, 2018).

Estudos recentes têm buscado analisar o comportamento dos usuários com base nos antecedentes da adoção da nova tecnologia (Lopes, Caracciolo, \& Herrero, 2018; Zhou, Lu, \& Wang, 2010) em algumas frentes como no efeito da confiança na intenção de uso (Lin, 2011; Liu, Min, \& Ji, 2009; Ramos et al., 2018), em determinantes de uso do mobile banking (Abbas et al., 2018; Ferreira \& Prearo, 2018). 
Além disso, estudos também investigaram aspectos demográficos, como o impacto de diferenças de gênero (Chawla, \& Joshi, 2018; Glavee-Geo, Shaikh \& Karjaluoto, 2017; Riquelme \& Rios, 2010; Wang, \& Petrounias, 2017), idade (Berraies, Ben Yahia, \& Hannachi, 2017; Chawla, \& Joshi, 2018; Choudrie et al., 2018; Krishanan, Teng, \& Khalidah, 2017; Koenig-Lewis, Palmer, \& Moll, 2010; Wang, \& Petrounias, 2017), nível de escolaridade (Agwu, \& Carter, 2018; Krishanan, Teng, \& Khalidah, 2017; Wang, \& Petrounias, 2017), renda (Chawla, \& Joshi, 2018; Wang, \& Petrounias, 2017), diferenças da adoção em áreas urbanas e metropolitanas (Gupta et al., 2017), local de moradia urbano ou rural (Suresh \& Namdeo, 2018) e em diferentes países e culturas (Laukkanen, \& Cruz, 2012).

Apesar desses estudos, a literatura ainda permanece muito incipiente e os resultados não são conclusivos devido ao uso de diferentes modelos e abordagens que não permitem generalização (Püschel, Mazzon, \& Hernandez, 2010; Shaikh, \& Karjaluoto, 2015).

Uma das lacunas que permanece em aberto diz respeito à utilização do serviço de MB para transações que envolvam movimentação financeira. Nesse sentido, esse estudo busca responder a seguinte questão de pesquisa: quais os fatores de maior impacto no uso do mobile banking para transações financeiras? Desse modo, o objetivo proposto é buscar identificar que fatores apresentam maior impacto no uso do mobile banking para movimentações financeiras como pagamentos, transferências, dentre outros.

Esse estudo avança em relação aos estudos anteriores ao analisar as características dos usuários do serviço de $\mathrm{MB}$ para movimentações financeiras ao invés de somente avaliar a intenção ou uso (não financeiro) do serviço. Dessa forma, atende aos chamados de Shaikh e Karjaluoto (2015) e Wang e Petrounias (2017) de se realizar um estudo com dados que contemplam diferentes regiões e características demográficas dos usuários em cada uma delas. Também atende a sugestão de Shaikh e Karjaluoto (2015) para se verificar se o MB é apenas um novo canal de acesso para os serviços bancários já existentes ou se é um novo instrumento de pagamentos, ou ambos (Dahlberg et al., 2008).

Em relação à prática gerencial, o estudo busca elucidar os conhecimentos das instituições financeiras quanto ao público usuário do serviço de MB para transações financeiras, de modo que viabilize a construção de estratégias específicas para ampliar o uso do serviço considerando-se características específicas de cada público e região. Desse modo, espera-se contribuir para que os investimentos em tecnologia bancária ( $\mathrm{R} \$ 19,5$ bilhões no Brasil em 
2017) sejam mais alinhados para a prática e transformação econômica, social e ambiental (Yadav, \& Pathak, 2014).

\section{Revisão da literatura}

\subsection{Mobile banking}

É notável que nos últimos anos o crescimento de utilização dos serviços MB ganhou destaque e configura-se pelo uso de dispositivos móveis - smartphones e tablets como meio de acesso e concretização de transações bancárias (Barnes e Corbitt, 2003; Zarifopoulos \& Economides, 2008; Lopes, Caracciolo e Herrero, 2018).

Tal motivação segundo (Lin, 2011; Watson et al. 2012) está relacionada à vantagem relativa percebida, facilidade de uso, compatibilidade, competência e integridade, que influenciam de forma significante a atitude comportamental de adotar ou continuar a usar serviços bancários móveis.

Diante disso, adotou-se neste trabalho o pressuposto de que os serviços MB são uma inovação tecnológica, à medida que permite que os clientes efetuem transações bancárias em qualquer tempo e lugar e, se utilizem das facilidades inerentes aos dispositivos móveis (Laukkanen, 2007b).

Neste contexto, um fator chave a ser investigado e que contribui para o ambiente econômico e social, que inclui fornecedores de serviços bancários, os usuários e os stakeholders em geral, são as características das regiões e pessoas que utilizam os serviços móveis.

A esse respeito, o estudo anterior de Nysveen, Perdersen e Thorbjornsen (2012) relaciona quatro influências gerais que afetam a intenção de uso: influências atitudinais, influências motivacionais, pressão e controle percebido. Neste estudo, os autores verificam que fatores comportamentais como a expressividade, diversão, funcionalidade, facilidade de uso, pressões normativas, controle comportamental, atitude e intenção de uso, influenciam a predisposição dos indivíduos para usarem mobile services.

Nesta linha de estudos, o estudo de Aziz, Badrawy e Hussien (2014) mostra que a facilidade de uso percebida, a utilidade percebida, o custo e a necessidade de interação, afetam de forma importante o uso de caixas eletrônicos, internet banking e mobile-banking. No entanto, o risco percebido tem um efeito significativo apenas entre os usuários e não usuários de caixas eletrônicos e mobile banking.

Em outra frente de estudos junto à 3.582 clientes bancários, Laukkanen e Cruz, (2012) examinaram antecedentes do comportamento de adoção do MB na Finlândia e Portugal e, 
incluíram variáveis como o papel da cultura nacional, aspectos sócio-demográficos e características específicas do comportamento do consumidor e, descobriram que fatores como o individualismo, a orientação a longo prazo e a masculinidade são determinantes significativos da cultura nacional no que tange à adoção do MB.

No continente asiático, mais especificamente na índia, outro aspecto que chama a atenção para o uso de MB (Gupta, Yun e Kim, 2017) está relacionado aos níveis de segurança e percepção de risco/controle associado aos serviços móveis, sobretudo em regiões urbanas. Corrobora neste sentido, o estudo de Min e Ji (2009), que apontam que a confiança funciona como um antecedente indireto da intenção do usuário em adotar o MB por meio de seu impacto sobre a utilidade percebida, e a forma geral da confiança do indivíduo é afetada principalmente pelas dimensões de confiança na tecnologia, confiança nos fornecedores e garantia estrutural.

Embora, sejam notórios alguns benefícios do uso de MB, em solo brasileiro verifica-se certas resistências que se contrapõe às expectativas da indústria. Um deles segundo Ramos, Ferreira e Rodrigues (2018) é inerente ao fator confiança que apresentou relação positiva com a intenção de uso de aplicativos bancários e, ainda tal relação foi influenciada pela facilidade de uso percebida, utilidade percebida, segurança, privacidade e familiaridade.

Em síntese, a literatura de adoção do MB é fragmentada, geralmente é baseada no modelo de aceitação da tecnologia e modificações e as variáveis de compatibilidade (estilo de vida e equipamentos), em que a utilidade percebida e a atitude são os impulsionadores mais significantes da intenção de serviços MB (Shaikh \& Karjaluoto, 2015). Os estudos anteriores não contemplam análises das regiões e características daqueles que já são usuários do serviço para movimentações financeiras e ainda analisam fatores de forma isolada e não conjunta.

\subsection{Determinantes do uso}

A análise da relação entre clientes e bancos através do MB não pode se limitar apenas à questão do desejo do cliente ou não de fazer uso de tecnologias, mas principalmente, deve-se considerar uma grande indústria que fomenta elevadas quantias de dinheiro e contribui para a economia dos países, portanto, detém recursos para pesquisar e buscar também respostas para efeitos comportamentais e sociais do uso dessas ferramentas.

Neste contexto, ainda que exista problemas de adaptação à tecnologias entre as gerações, que podem comprometer a confiança, a lealdade e a satisfação (Berraies, Yahia e 
Hannachi, 2017), faz-se necessário ações mais objetivas para tornar as tecnologias mais acessíveis e agradáveis a todos os usuários.

Portanto, questões demográficas devem ser consideradas quando busca-se conhecer os determinantes do uso, em razão de que, por exemplo, o uso que um jovem da Geração Z faz de tecnologias digitais não é o mesmo que um idoso consegue fazer. Diante disso o imperativo para o uso destas tecnologias, que segundo Abbas, Hassan, Asif, Junaid, Zainab (2018) está centrado no autoatendimento e ganho de tempo, pode não ter a mesma função utilidade nas gerações anterior à década de 1980.

Além disso, Wang e Petrounias (2017) enfatizam que as variáveis demográficas são importantes porque representam um caminho para o desenvolvimento de estratégias de marketing, porém, os fatores demográficos que podem ter influência significativa sobre os serviços bancários não foram estudados com profundidade suficiente e a maioria dos estudos que investigam a adoção de tecnologias ignora os fatores demográficos como renda, idade, educação, experiência bancária e gênero (Crabbe et al, 2009).

Contudo, é inegável que as tecnologias digitais ocupam espaço cada vez mais amplo nos serviços bancários e, diante disso, torna-se importante conhecer quais os principais determinantes do uso de tais tecnologias bancárias pelos indivíduos. De forma geral, Abbas et al (2018) segregam tais determinantes em quatro fatores, a saber: facilidade de uso percebida, utilidade percebida, risco percebido, influência social, vantagem relativa e confiança percebida.

Neste contexto, as variáveis demográficas são investigadas por apresentarem um efeito moderador que influencia o usuário em sua atitude em relação aos serviços bancários (Chawla e Joshi, 2018) e as mais exploradas são gênero, idade, renda e formação educacional.

\subsubsection{Renda}

De forma geral a renda está ligada ao dinheiro ganho por indivíduos em troca do fornecimento de produtos ou serviços, portanto, pode influenciar o comportamento e a atitude dos indivíduos em relação ao acesso as tecnologias. O estudo de Porter e Donthu (2006) mostra que consumidores de baixa renda são mais preocupados com custos e apresentam a percepção de baixa utilidade percebida quando estão diante de produtos tecnológicos de alto custo, ao contrário os consumidores com maior renda são capazes de pagar preços mais altos pela tecnologia.

Outro aspecto importante a ser destacado é o nível de ansiedade presente na utilização de tecnologias. Isto se torna mais claro a partir dos resultados de um estudo de Lee et al. (2010) 
que descobriram que a ansiedade durante a utilização da tecnologia diminui à medida que o nível de renda aumenta.

Destaca-se também a linha de estudos relacionada ao estilo de vida dos clientes bancários, que pode ser um fator determinante na escolha do serviço bancário. Fino (2015) apresenta quatro seguimentos que apresentam diferentes características de adoção de tecnologias, i) os positivos e compradores online, que principalmente utilizam a internet para comprar produtos/serviços online, ii) os fãs das redes sociais, que consideram que a internet é uma ferramenta que proporciona interação entre as pessoas, iii) os curiosos e sensíveis que acreditam nos benefícios da Internet, que gostam de utilizar a última tecnologia e acreditam nos benefícios da Internet e, por último, iv) os informados através da Internet, que consideram que a internet proporciona um ambiente de aprendizagem.

Em linha com os pressupostos desta pesquisa, o modelo AIO adotado no estudo de Fino (2015) para definir o estilo de vida, apresenta uma relação direta com características demográficas e, dentre elas a renda, que representa um dos determinantes do estilo de vida que remete a maior ou menor uso de tecnologias bancárias.

Partindo-se da motivação de investigar o efeito da variável renda sobre a decisão de utilização dos serviços mobile banking no Brasil, levanta-se a seguinte hipótese de pesquisa:

H1: Indivíduos com nível de renda mais alta utilizam os serviços mobile banking para movimentação financeira com maior frequência.

\subsubsection{Escolaridade}

Os estudos mais antigos da literatura que pesquisaram a escolaridade, geralmente apontam que usuários de tecnologia com baixa escolaridade percebem grandes barreiras ao uso da internet e do comércio eletrônico em comparação com usuários com alto nível educacional (Liebermann e Stashevsky, 2002). Neste sentido, o estudo de Ferreira e Prearo (2018) mostra que, quanto mais elevado o nível de escolaridade do cliente bancário, maior a propensão dele consumir produtos e serviços bancários digitais.

Porém, a literatura mais atual apresenta resultados destoantes, como no estudo de Chawla e Joshi (2018), em que a variável escolaridade não apresentou efeito moderador sobre o uso de MB. Tal fato pode estar relacionado ao fato que, pessoas expostas a níveis mais altos de educação provavelmente tiveram mais exposição à tecnologia de forma precoce, não apenas em 
seu local de trabalho, mas também em cursos, treinamentos e atividades do dia-a-dia (Weijters et al., 2007).

Contudo, a variável demográfica educação tem sido investigada em estudos internacionais, como aponta o estudo de Shaikh e Karjaluoto (2015), no qual o nível de escolaridade exerce um efeito significativo sobre a adoção de MB. Corroboram neste sentido Pimenta e Ramos (2010) que consideram que a educação exerce um efeito moderador do nível de escolaridade frente às dificuldades na utilização dos terminais de autoatendimento, assim, os idosos mais escolarizados sentem-se relativamente mais seguros, confiantes, menos ansiosos e nervosos, bem como possuem mais capacidade cognitiva de compreensão no processo de interação de autoatendimento bancário.

Neste contexto, a hipótese $\mathrm{H} 2$ busca investigar a relação entre o nível de escolaridade dos indivíduos e o nível de uso de serviços bancários digitais por meio de dispositivos móveis.

H2: Indivíduos com nível de escolaridade mais alta utilizam os serviços mobile banking para movimentações financeiras com maior frequência.

\section{Procedimentos metodológicos}

Para atingir o objetivo proposto e testar as hipóteses, realizou-se uma pesquisa descritiva de abordagem quantitativa, caracterizada como um estudo de caso único que, segundo Yin (2015), pode ser usado para contribuir para o conhecimento dos fenômenos individuais, grupais, organizacionais, sociais, políticos e relacionados. Esse método permite ao pesquisador focar em um caso e obter uma perspectiva útil para as demais organizações.

\subsection{População e amostra}

O país de realização do estudo foi o Brasil, por fazer parte dos BRIC's, ser considerado uma economia emergente e apresentar um dos sistemas bancários mais desenvolvidos do mundo (Febraban, 2018). A delimitação geográfica é representada pelo Estado do Rio Grande do Sul, por ser o Estado que apresenta maior percentual da população com telefone móvel celular para uso pessoal (IBGE, 2018) além de apresentar regiões urbanas, rurais e metropolitanas.

A empresa alvo do estudo é uma instituição financeira estruturada em 1169 pontos de atendimento, em que dois estão no exterior e o restante abrangem sete Estados brasileiros com um total de 11.050 colaboradores. Possui filiais em aproximadamente $97 \%$ dos municípios do Estado e foi escolhida por conveniência e por apresentar características que estão relacionadas 
ao objetivo do estudo. A amostra final consiste em dados de 338 municípios do Estado que continham agências bancárias da instituição e que, possuíam histórico de transações em canais digitais no período analisado.

\subsection{Coleta e tratamento dos dados}

Foram realizadas cinco visitas ao centro administrativo da empresa e duas entrevistas com o executivo responsável pela área comercial no período de 01 a 31 de outubro de 2017, para a coleta dos dados referente às transações em mobile banking que envolvem movimentação financeira. Os dados demográficos referentes ao PIB per capita dos municípios e o índice de Gini foram obtidos junto à página do IBGE (www.ibge.gov.br) e os dados referentes à população e escolaridade dos maiores de 18 anos foram obtidos junto a página do Tribunal Superior Eleitoral - TSE (www.tse.jus.br).

Em seguida, foram tabulados todos os dados coletados: PIB per capita, índice de Gini, níveis de escolaridade (Analfabeto, Lê e escreve, Ensino Fundamental Incompleto, Ensino Fundamental Completo, Ensino Médio Incompleto, Ensino Médio Completo, Ensino Superior Incompleto e Ensino Superior Completo) e as transações em canais digitais realizadas em cada município de atuação da instituição financeira alvo do estudo mês a mês (março 2017 a agosto 2018).

\subsection{Procedimento de análise dos dados}

Para responder o problema de pesquisa e testar as hipóteses propostas, realizou-se regressões lineares para verificar os efeitos do PIB per capita, Índice de Gini e Escolaridade, isolados e em conjunto, por meio do software Statistical Package for the Social Sciences $(S P S S)$, versão $23 \circledR$. Utilizou-se a regressão linear por possibilitar o estudo da relação entre uma ou mais variáveis explicativas, que se apresentam na forma linear e uma variável dependente quantitativa (Fávero \& Fávero, 2016). O objetivo desse procedimento foi de mensurar o quanto cada uma dessas variáveis independentes influencia no desempenho dos gestores. Desse modo, o modelo de regressão linear utilizado é descrito na forma da equação (1):

UsoMB $_{t i}=\beta_{0 i}+\beta_{1} P I B+\beta_{2}$ Gini $_{i}+\beta_{3}$ EnsSup $_{i}+\beta_{4}$ EnsSupInc $_{i}+\beta_{5}$ EnsMedC $_{i}+$ $\beta_{6}$ EnsMedInc $_{i}+\beta_{67}$ EnsFunC $_{i}+\beta_{8}$ EnsFunInc $_{i}+\beta_{9}$ LeEscreve $+\beta_{10}$ Analfabeto $+\epsilon_{i}$ 
Em que:

UsoMB: representa o uso efetivo de mobile banking para movimentações financeiras no banco em estudo a cada mês.

PIB: representa o PIB per capita nos municípios do estudo.

Gini: representa o indicador de concentração de renda no município.

EnsSupC: população com Ensino Superior Completo.

EnsSupInc: população com Ensino Superior Incompleto.

EnsMedC: população com Ensino Médio Completo.

EnsMedInc: população com Ensino Médio Incompleto.

EnsFunC: população com Ensino Fundamental Completo.

EnsFunInc: população com Ensino Fundamental Incompleto.

LeEscreve: população que apenas lê e escreve.

Analfabeto: população que não sabe ler nem escrever.

$i$ : amostra específica de município.

$\epsilon$ : erro amostral.

Na etapa de análise dos dados, serão apresentados o resultado das regressões, a análise da aceitação ou rejeição das hipóteses da pesquisa e a discussão dos resultados obtidos neste estudo frente a outras pesquisas.

\section{Apresentação dos resultados}

Inicialmente, buscou-se analisar a evolução do uso do MB para movimentação financeira na instituição em estudo. Para isso, utilizou-se a soma do número de movimentações por esse canal nos 338 municípios da amostra no período março 2017 a agosto 2018. Os resultados são apresentados na Figura 1.

Considerando-se um período de 12 meses a partir de maio de 2017, observa-se maior intensidade de uso, que culmina com um total de movimentações 45 vezes maior que em abril 2018, o que representa um crescimento superior à média brasileira (Febrabran, 2018). Em contrapartida, ao comparar-se o crescimento no $2^{\circ}$ semestre de 2017 (381\%) com o do $1^{\circ}$ semestre de 2018 (30\%) e com o último trimestre do período da amostra (10\%), é possível identificar uma forte desaceleração no avanço do uso para movimentações financeiras.

Uma das causas possíveis para esse fato é que, a população com maior propensão ao uso, começou a utilizar o serviço em maior intensidade após seu lançamento em março 2017. No período final da amostra, não existe mais demanda represada inicialmente e o crescimento da utilização está ocorrendo de acordo com a venda do serviço e em proporção semelhante ao dos demais produtos e serviços oferecidos pela instituição. 


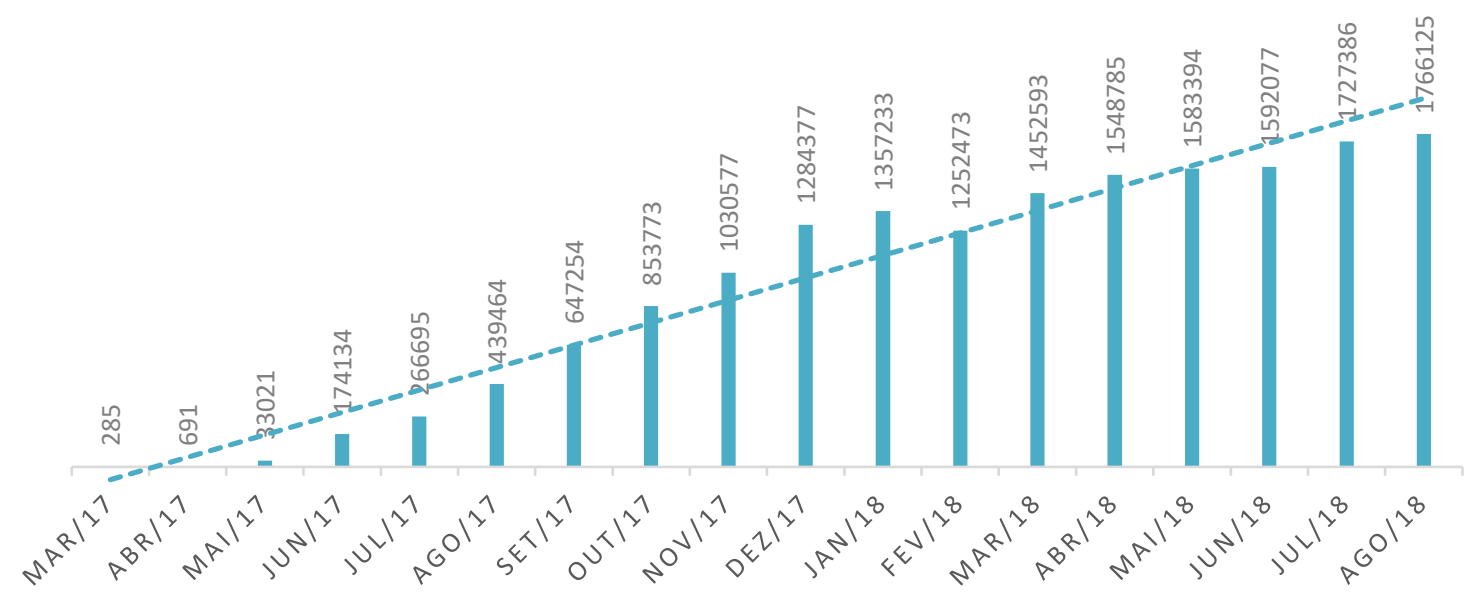

Figura 1: Evolução da movimentação financeira através de Mobile Banking nos municípios da amostra

Fonte: Dados da pesquisa.

Em seguida, realizou-se as regressões lineares e, tendo em vista que a coleta obteve dados de março 2017 até agosto 2018, optou-se por analisar as relações entre as variáveis em 3 meses específicos desse período, julho e dezembro 2017 e junho 2018, por representarem o período de 1 ano e permitir comparações com resultados de outros estudos que analisaram o mesmo período.

Os primeiros resultados das três regressões mostrados na Tabela 1 apontam que, a renda per capita e o índice de Gini não apresentaram efeito significante sobre a uso dos canais digitais. Considerando-se que, o índice de Gini aponta a diferença de renda entre os mais pobres e os mais ricos e varia de zero (sem desigualdade, ou seja, todos com a mesma renda) e 1 (somente uma pessoa detém toda a riqueza), não é possível afirmar que municípios com maior renda apresentam maior volume de movimentações financeiras via mobile banking. Esses resultados rejeitam a hipótese $\mathrm{H}_{1}$ desse estudo e apresentam novas considerações acerca da variável, portanto, diverge dos estudos de Porter e Donthu (2006) e Fino (2015).

Uma possível causa dessa divergência nos resultados é o fato de que tais autores analisaram somente intenção de uso e não o uso efetivo. Além disso, o estudo foi realizado no estado do Rio Grande do Sul que apresentou em 2017 um Índice de Gini de apenas 0,492 (IBGE, 2017), além de fazer parte da Região Sul, que apresenta o menor índice do Brasil. 
Portanto, a variância da distribuição de renda entre os municípios é baixa e indica que a renda não é fator explicativo para as diferentes quantidades de utilização de canais digitais como o MB para as movimentações financeiras nas cidades da amostra.

\section{Tabela 1}

Resultados das regressões lineares com adoção das movimentações via MB dos meses de julho 2017, dezembro 2017 e junho 2018 como variável independente

\begin{tabular}{|c|c|c|c|c|c|c|c|c|c|}
\hline \multirow{2}{*}{$\begin{array}{c}\text { Variável } \\
\text { Dependente: }\end{array}$} & \multicolumn{3}{|c|}{ Julho 2017 } & \multicolumn{3}{|c|}{ Dezembro 2017} & \multicolumn{3}{|c|}{ Junho 2018} \\
\hline & $\begin{array}{c}\text { Coef. } \\
\text { Padronizado }\end{array}$ & Teste $\mathbf{t}$ & Sig. & \begin{tabular}{|c|c|} 
Coef. \\
Padronizado
\end{tabular} & Teste $\mathbf{t}$ & Sig. & $\begin{array}{c}\text { Coef. } \\
\text { Padronizado }\end{array}$ & Teste $\mathbf{t}$ & Sig. \\
\hline PIB & ,000 & 015 & ,988 &,- 001 &,- 157 &, 875 &,- 001 &,- 163 & ,871 \\
\hline Gini &,- 007 & -972 & ,332 &,- 006 &,- 859 & ,391 &,- 006 &,- 897 & ,371 \\
\hline EnsSupC & , 138 & 1,943 & ,053 & ,152 & 2,258 & ,025 & , 179 & 2,790 & ,006 \\
\hline EnsSupInc & 1,040 & 9,531 & ,000 & ,985 & 9,492 & ,000 & ,913 & 9,217 &, 000 \\
\hline EnsMedC &, 065 & ,949 & 343 & 109 & 1,679 & ,094 &, 113 & 1,812 & ,071 \\
\hline EnsMedInc & 1,145 & 29,838 & ,000 & 1,114 & 30,534 & ,000 & 1,092 & 31,355 &, 000 \\
\hline EnsFunC & $-1,016$ & $-11,110$ & ,000 & $-1,014$ & $-11,669$ & ,000 &,- 963 & $-11,615$ & ,000 \\
\hline EnsFunInc &,- 485 & $-9,091$ & ,000 &,- 460 & $-9,074$ & ,000 &,- 443 & $-9,158$ &, 000 \\
\hline LeEscreve & 0,36 & 1,149 & 251 & 027 & 917 & 360 & ,023 &, 824 & ,411 \\
\hline Analfabeto & 0,26 & ,912 & ,363 & ,043 & 1,576 &, 116 & 048 & 1,849 &, 065 \\
\hline Controle Fixo & \multicolumn{3}{|c|}{ Sim } & \multicolumn{3}{|c|}{ Sim } & \multicolumn{3}{|c|}{ Sim } \\
\hline $\mathrm{R}^{2}$ & \multicolumn{3}{|c|}{0,985} & \multicolumn{3}{|c|}{0,986} & \multicolumn{3}{|c|}{0,987} \\
\hline $\mathrm{R}^{2}$ ajustado & \multicolumn{3}{|c|}{0,984} & \multicolumn{3}{|c|}{0,986} & \multicolumn{3}{|c|}{0,987} \\
\hline ANOVA & \multicolumn{3}{|c|}{2081,804} & \multicolumn{3}{|c|}{2309,303} & \multicolumn{3}{|c|}{2538,174} \\
\hline Durbin-Watson & \multicolumn{3}{|c|}{2,185} & \multicolumn{3}{|c|}{2,125} & \multicolumn{3}{|c|}{2,101} \\
\hline $\mathrm{N}^{\mathrm{o}}$ Observações & \multicolumn{3}{|c|}{338} & \multicolumn{3}{|c|}{338} & \multicolumn{3}{|c|}{338} \\
\hline
\end{tabular}

Fonte: dados da pesquisa

Por outro lado, em ambos os períodos analisados, a escolaridade demonstrou impactar o uso de MB para movimentações financeiras e isto confirma a hipótese $\mathrm{H}_{2}$ desse estudo. De todos os níveis de escolaridade analisados, somente o "Ensino Médio Completo", "Lê e Escreve" e o "Analfabeto" não apresentaram resultados significantes para o uso dos canais digitais.

Baixos níveis de escolaridade mostraram resultados negativos e significativos nos três períodos como no caso do Ensino Fundamental Completo $(-1,016 ;-1,014$ e -0,963, p<0,01) e Incompleto $(-0,485 ;-0,460$ e $-0,443, p<0,01)$. Esses resultados permitem concluir que a baixa formação escolar em níveis inferiores influencia negativamente no uso do MB para movimentações financeiras. Além disso, esses resultados confirmam achados da literatura em relação a intenção de uso, que apontam que o menor conhecimento sobre o serviço gera insegurança, medo e outros sentimentos de aversão ao uso (Liebermann e Stashevsky, 2002).

Em relação aos maiores níveis de escolaridade que apresentaram resultados significativos e positivos, pode-se inferir que indivíduos com níveis mais altos de formação utilizam mais o serviço em relação a indivíduos de níveis mais baixos. Porém, essa relação não 
é válida como um todo, pois tanto o Ensino Superior Incompleto $(1,040 ; 0,985$ e 0,913,p< $<0,01)$ quanto o Ensino Médio Incompleto $(1,145 ; 1,114$ e 1,092, p<0,01) apresentaram maior impacto sobre as variáveis independentes do que estes níveis de ensino com formação completa.

Esses achados, apesar de confirmarem a literatura anterior no que concerne a comparação a níveis mais baixos como Ensino Fundamental ou Lê e Escreve, demonstram que outras variáveis contextuais também podem também ter poder explicativo tanto sobre a intensidade de uso do MB quanto sobre o impacto do nível de escolaridade sobre tal fator.

\section{Discussões}

Um dos resultados importantes observados neste trabalho remete à variável demográfica nível de escolaridade, em que se verifica que o nível superior incompleto e o ensino médio incompleto apresentaram níveis de significância maiores do que os mesmos níveis completos, ou seja, assim como no estudo de Braga, Barboza, \& Bouzada (2017), há indicativo de que outras variáveis podem estar afetando a percepção dos usuários, além do fator educação.

Embora a variável idade não tenha sido objeto específico das análises, é possível que o fator idade exerça intervenção sobre o nível de percepção dos usuários e, em conjunto com o fator educação possam ter influência nos resultados finais. A esse respeito, o estudo de Pimenta \& Ramos (2010) constataram que o idoso com nível educacional mais baixo tenderá a ser mais resistente à adoção de terminais de auto atendimento e, logo por analogia pode ocorrer também no uso MB, o que ocasionará mais dependência de terceiros, aumentará o nível de insegurança, angústia e medo.

Ao olhar para o fator renda, nos últimos se verifica que o serviços bancários eletrônicos deixaram de ser um privilégio para as camadas sociais mais altas e houve uma mudança de paradigma na distribuição dos serviços para o público de baixa renda, por meio da chamada quinta onda (Cernev, Diniz, \& Jayo, 2009), por este motivo atualmente o fator renda não impacta negativamente na utilização dos serviços MB.

Decorrente das políticas econômicas voltadas à melhor distribuição de renda entre as classes sociais no Brasil, Bader \& Savoia (2013) explicam que ocorre uma maior capilaridade das empresas do setor financeiro, menores custos e orientação para as necessidades dos clientes, e decorrente disso os serviços bancários deverão ser adequados ao universo de inclusão financeira. 


\section{Considerações finais}

Este estudo teve por objetivo identificar que fatores apresentam maior impacto no uso do mobile banking para movimentações financeiras como pagamentos, transferências, dentre outros, e aponta, de forma geral, que maiores índices de uso de MB estão relacionados de forma significante a níveis de escolaridade mais altos, o que confirma o resultado de outros estudos da literatura nacional e internacional.

Diante disso, pode-se afirmar que, o diferencial deste trabalho é a utilização de fatores demográficos mostrados na literatura como uma carência da pesquisa na área (Wang e Petrounias, 2017; Crabbe et al. 2009).

Limitações quanto a generalização teórica estão relacionadas ao estudo com área geográfica limitada e as próprias características demográficas e culturais do Estado do Rio Grande do Sul.

Outra limitação é a quantidade de variáveis adotadas nesta pesquisa, que embora preencham uma lacuna de pesquisa requerida na literatura, pode ser expandida em novos estudos, com a inclusão de outras variáveis demográficas e o aprofundamento na questão do autocontrole do comportamento de consumo, que enquanto variável comportamental pode também afetar as decisões de aquisição de tecnologias.

Para futuras pesquisas, sugere-se aprofundar-se na variável educação, contudo, segregando-se em educação privada e particular, ensino técnico e normal. Além disso, o fator idade, não abordado neste estudo, pode também ser uma variável que exerça impacto no uso do mobile banking, haja vista, representar estímulos diferentes para o uso do serviço MB.

\section{Referências}

Abbas, S. K.; Hassan, H. A.; Asif, J.; Junaid, H. M.; \& Zainab, F. (2018). What are the key determinants of mobile banking adoption in Pakistan? International Journal of Scientific \& Engineering Research, 9(2), 840-848. DOI: 10.14299/ijser.2018.02.012

Agwu, E.; \& Carter, A. L. (2018). Mobile phone banking in Nigeria: benefits, problems and prospects. International Journal of Business and Commerce, 3(6), 50-70. Retrieved from https://papers.ssrn.com/sol3/papers.cfm?abstract_id=3120495

Aziz, R. A. E.; ElBadrawy, R.; \& Hussien, M. I. (2014). ATM, internet banking and mobile banking services in a digital environment: the Egyptian banking industry. International 
Journal of Computer Applications, 90(8), 45-52. Retrieved from http://citeseerx.ist.psu.edu/viewdoc/summary?doi=10.1.1.683.9637

Bader, M.; \& Savoia, J. R. F. (2013). Logística da distribuição bancária: tendências, oportunidades e fatores para inclusão financeira. Revista de Administração de Empresas, 53(2), 208-2015. DOI: http://dx.doi.org/10.1590/S0034-75902013000200008

Barnes, S. J.; \& Corbitt, B. (2003) Mobile banking: concept and potential. International Journal of Mobile Communications, 1(3), 273-288. DOI: 10.1504/IJMC.2003.003494

Berraies, S.; Yahia, K. B; \& Hannachi, M. (2017). Identifying the effects of perceived values of mobile banking applications on customers: Comparative study between baby boomers, generation X and generation Y. International Journal of Bank Marketing, 35(6), 10181038. DOI: https://doi.org/10.1108/IJBM-09-2016-0137

Braga, B. S.; Barbosa, J. G. P.; \& Bouzada, M. A. C. (2017). A influência de características demográficas dos usuários sobre sua decisão de adoção do mobile banking. Revista Brasileira de Pesquisas de Marketing, Opinião e Mídia, 10(2), 136-153. Recuperado de http://www.revistapmkt.com.br/pt-

br/anteriores/anteriores.aspx?udt_863_param_detail=8648

Brown, I.; Cajee, Z.; Davies, D.; \& Stroebel, S. (2003). Cell phone banking: predictors of adoption in South Africa: an exploratory study. International journal of information management, 23(5), 381-394. DOI: https://doi.org/10.1016/S0268-4012(03)00065-3

Carlsson, C.; Walden, P.; \& Bouwman, H. (2006). Adoption of 3G+ services in Finland. International Journal of Mobile Communications, 4(4), 369-385. DOI: https://doi.org/10.1504/IJMC.2006.008947

Cernev, A; Diniz, E.; \& Jayo, M. (2009). Emergência da quinta onda de inovação bancária. Anais... Americas Conference on Information Systems (AMCIS). Proceedings. 4. Recuperado https://aisel.aisnet.org/cgi/viewcontent.cgi?article=1079\&context=amcis2009

Chawla, D.: \& Joshi, H. (2018). The Moderating Effect of Demographic Variables on Mobile Banking Adoption: An Empirical Investigation. Global Business Review, 19(3), 90-113. DOI: https://doi.org/10.1177/0972150918757883

Coelho, F.; \& Easingwood, C. (2003). Multiple channel structures in financial services: a framework. Journal of Financial Services Marketing, 8(1), 22-34. DOI: https://doi.org/10.1057/palgrave.fsm.4770104

Choudrie, J.; Junior, C. O.; McKenna, B.; \& Richter, S. (2018). Understanding and conceptualising the adoption, use and diffusion of mobile banking in older adults: A research agenda and conceptual framework. Journal of Business Research, 88, 449-465. DOI: https://doi.org/10.1016/j.jbusres.2017.11.029 
Crabbe, M.; Standing, C.; Standing, S.; \& Karjaluoto, H. (2009). An adoption model for mobile banking in Ghana. International Journal of Mobile Communications, 7(5), p. 515-543 DOI: 10.1504/IJMC.2009.024391

Dahlberg, T.; Mallat, N.; Ondrus, J.; \& Zmijewska, A. (2008). Past, present and future of mobile payments research: A literature review. Electronic Commerce Research and Applications, 7(2), 165-181. DOI: https://doi.org/10.1016/j.elerap.2007.02.001

Fávero, L. P.; \& Fávero, P. (2016). Análise de dados: modelos de regressão com Excel®, Stata® e SPSS®, v.1. Campus: Elsevier Brasil.

Febraban. Federação Brasileira de Bancos. (2018). Pesquisa FEBRABAN de Tecnologia Bancária 2018. Deloitte. Recuperado de https://cmsportal.febraban.org.br/Arquivos/documentos/PDF/febraban_2018_Final.pdf

Federal Reserve System. (2016). Consumers and Mobile Financial Services 2016. Board of Governors of the Federal Reserve System. Recuperado de https://www.federalreserve.gov/econresdata/consumers-and-mobile-financial-servicesreport-201603.pdf

Ferreira, R., M.; \& Prearo, L. C. (2018). Determinantes no uso do mobile banking para operações e transações financeiras em dispositivos móveis: um estudo financeiro no município de São Paulo. Navus-Revista de Gestão e Tecnologia, 8(3), 23-36. DOI: http://dx.doi.org/10.22279/navus.2018.v8n3.p.23-36.654

Fino, P. S. (2015). Dissertação (Mestrado em Marketing). A influência do estilo de vida do cliente na escolha dos canais e serviços bancários. Lisbon School of Economics \& Management - Universidade de Lisboa, Lisboa-PT. Retrieved from https://www.repository.utl.pt/bitstream/10400.5/10985/1/DM-PSF-2015.pdf

Funk, J. L. (2009). The emerging value network in the mobile phone industry: The case of Japan and its implications for the rest of the world. Telecommunications Policy, 33(1-2), 4-18. DOI: https://doi.org/10.1016/j.telpol.2008.09.002

Glavee-Geo, R., Shaikh, A. A., \& Karjaluoto, H. (2017). Mobile banking services adoption in Pakistan: are there gender differences? International Journal of Bank Marketing, 35(7), 1090-1114. DOI: https://doi.org/10.1108/IJBM-09-2015-0142

Gupta, S.; Yun, H.; Xu, H.; \& Kim, H. W. (2017). An exploratory study on mobile banking adoption in Indian metropolitan and urban areas: a scenario-based experiment. Information Technology for Development, 23(1), 127-152. DOI: https://doi.org/10.1080/02681102.2016.1233855

Instituto Brasileiro de Geografia e Estatística - IBGE. (2014). Programa Nacional Por Amostra de Domicílios - $P N A D$. Recuperado de https://ww2.ibge.gov.br/home/estatistica/populacao/acessoainternet2014/default.shtm

Instituto Brasileiro de Geografia e Estatística - IBGE. (2018). Pesquisa Nacional por Amostra de Domicílios Contínua - PNAD Contínua. Acesso à Internet e à Televisão e Posse de 
Telefone Móvel Celular para Uso Pessoal. Recuperado de ftp://ftp.ibge.gov.br/Trabalho_e_Rendimento/Pesquisa_Nacional_por_Amostra_de_Domi cilios_continua/Anual/Acesso_Internet_Televisao_e_Posse_Telefone_Movel_2016/Anali se_dos_Resultados.pdf.

Krishanan, D.; Teng, K. L. L.; \& Khalidah, S. (2017). Moderating effects of age \& education on consumers' perceived interactivity \& intention to use mobile banking in Malaysia: A structural equation modeling approach. Anais... Proceeding of International Conference on Humanities, Language, Culture \& Business, Penang 2017, Hotel Bayview Beach Resort Batu Feringgi Penang Malaysia.

Koenig-Lewis, N.; Palmer, A.; \& Moll, A. (2010). Predicting young consumers' take up of mobile banking services. International journal of bank marketing, 28(5), 410-432. DOI: https://doi.org/10.1108/02652321011064917

Laukkanen, T. (2007). Internet vs mobile banking: comparing customer value perceptions. Business Process Management Journal, 13(6), 788-797. DOI: https://doi.org/10.1108/14637150710834550

Laukkanen, T. (2007b). Measuring mobile banking customers' channel attribute preferences in service consumption. International Journal of Mobile Communications, 5(2), 123-138. DOI: 10.1504/IJMC.2007.011812

Laukkanen, T.; \& Cruz, P. (2012). Cultural, individual and device-specific antecedents on mobile banking adoption: a cross-national study. Anais... 45th Hawaii International Conference on System Sciences, 3170-3179. Maui, HI, USA. IEEE. DOI: 10.1109/HICSS.2012.189

Lin, H. F. (2011). An empirical investigation of mobile banking adoption: The effect of innovation attributes and knowledge-based trust. International journal of information management, 31(3), 252-260. DOI: https://doi.org/10.1016/j.ijinfomgt.2010.07.006

Liu, Z.; Min, Q.; \& Ji, S. (2009). An empirical study on mobile banking adoption: The role of trust. Anais... Second International Symposium on Electronic Commerce and Security, 2009. v. 2, 7-13, Nanchang, China. IEEE. DOI: 10.1109/ISECS.2009.150

Lopes, E. L.; Caracciolo, L. L.; \& Herrero, E. (2018). A Aceitação do mobile banking no Brasil: uma Análise por meio do Modelo TAM Estendido. TPA-Teoria e Prática em Administração, 8(1), 190-221. DOI: http://dx.doi.org/10.21714/2238-104X2018v8i137816

Nysveen, H.; Pedersen, P. E.; \& Thorbjørnsen, H. (2012). Intentions to use mobile services: Antecedents and cross-service comparisons. Journal of the Academy of Marketing Science, 33(3), 330-346. DOI: https://doi.org/10.1177/0092070305276149

Pimenta, I. L.; \& Ramos, A. S. M. (2010). Efeito moderador do nível de escolaridade sobre as dificuldades e barreiras na utilização dos terminais de autoatendimento bancário entre 
os idosos. Revista Movendo Ideias, 17(2), 19-39. DOI: http://dx.doi.org/10.17800/movendo\%20ideias.v15i2.563

Pousttchi, K.; \& Schurig, M. (2004). Assessment of today's mobile banking applications from the view of customer requirements. Anais... Proceedings of 37th Annual Hawaii International Conference on System Sciences. IEEE. Big Island, HI, USA. DOI: 10.1109/HICSS.2004.1265440

Püschel, J.; Mazzon, J. A; \& Hernandez, J. M. C. (2010). Mobile banking: proposition of an integrated adoption intention framework. International Journal of Bank Marketing, 28(5), 389-409. DOI: https://doi.org/10.1108/02652321011064908

Ramos, F. L.; Ferreira, J. B.; Freitas, A. S.; \& Rodrigues, J. W. (2018). The effect of trust in the intention to use m-banking. Brazilian Business Review, 15(2), 175-191. DOI: DOI:10.15728/bbr.2018.15.2.5

Riquelme, H. E.; \& Rios, R. E. (2010). The moderating effect of gender in the adoption of mobile banking. International Journal of Bank Marketing, 28(5), 328-341. DOI: https://doi.org/10.1108/02652321011064872

Shaikh, A. A.; Hanafizadeh, P.; \& Karjaluoto, H. (2017). Mobile banking and payment system: A conceptual standpoint. International Journal of E-Business Research (IJEBR), 13(2), 14-27. DOI: 10.4018/IJEBR.2017040102

Shaikh, A. A.; \& Karjaluoto, H. (2015). Mobile banking adoption: A literature review. Telematics and Informatics, 32(1), 129-142. DOI: https://doi.org/10.1016/j.tele.2014.05.003

Suresh, A. S.; \& Namdeo, V. (2018). A critical review: Mobile banking services and its significant impact on rural customer behaviour patterns. Anais... IJSRST, 4(2), 694-701. Recuperado de http://ijsrst.com/paper/2207.pdf

Wang, S.; \& Petrounias, I. (2017). Big data analysis on demographic characteristics of chinese mobile banking users. Anais... 19th Conference on Business Informatics (CBI) (pp. 4754). IEEE. Thessaloniki, Greece. DOI: 10.1109/CBI.2017.72

Wang, Y. S.; Lin, H. H.; \& Luarn, P. (2006). Predicting consumer intention to use mobile service. Information Systems Journal, 16(2), 157-179. $\quad$ DOI: https://doi.org/10.1111/j.1365-2575.2006.00213.x

Watson, Richard T.; Pitt, L. F.; Berthon, P.; \& Zinkhan, G. M.; (2002). U-commerce: Expanding the universe of marketing. Journal of the Academy of Marketing Science, 30(4), 333-347. DOI: https://doi.org/10.1177/009207002236909

Yadav, R.; \& Pathak, G. (2014). Environmental sustainability through green banking: A study on private and public sector banks in India. OIDA International Journal of Sustainable Development, 6(8), 37-48, 2013. Retrieved from SSRN: https://ssrn.com/abstract=2385573 
Zarifopoulos, M.; \& Economides, A. A. (2008). Evaluating mobile banking portals. International Journal of Mobile Communications, 7(1), 66-90. Retrieved from https://www.researchgate.net/profile/Anastasios_Economides/publication/220474633_Ev aluating_mobile_banking_portals/links/00b495295b518d4873000000.pdf

Zhou, T.; Lu, Y.; \& Wang, B. (2010). Integrating TTF and UTAUT to explain mobile banking user adoption. Computers in Human Behavior, 26(4), 760-767. DOI: https://doi.org/10.1016/j.chb.2010.01.013 